\title{
Spectro-morphology of galaxies: A multi-wavelength (UV-R) classification method
}

\author{
S. Lauger, D. Burgarella, and V. Buat
}

\author{
Laboratoire d'Astrophysique de Marseille, Traverse du Siphon BP 8, 13376 Marseille Cedex 12, France \\ e-mail: sebastien.lauger@oamp.fr
}

Received 24 January 2003 / Accepted 1 October 2004

\begin{abstract}
We present a quantitative method to classify galaxies, based on multi-wavelength data and constructed from the properties of nearby galaxies. Our objective is to define an classification method that can be used for low and high redshift objects. We estimate the concentration of light $(C)$ at the galaxy center and the $180^{\circ}$ rotational asymmetry $(A)$, computed at several wavelengths, from ultraviolet (UV) to $I$-band. The variation of the indices of concentration and asymmetry with the wavelength reflects the proportion and the distribution of young and old stellar populations in galaxies. In general $C$ is found to decrease, and $A$ to increase from optical to UV: the patchy appearance of a galaxy in the UV with no bulge is often very different from its counterpart at optical wavelengths, with a prominent bulge and a more regular disk. We quantify the variation of $C$ and $A$ with wavelength. In this way we are able to distinguish five types of galaxies that we call spectro-morphological types: compact, ringed, spiral, irregular and central-starburst galaxies, which can be differentiated by the distribution of their stellar populations. We discuss in detail the morphology of the galaxies of the sample, and describe the morphological characteristics of each spectro-morphological type. We apply spectro-morphology to three objects at a redshift $z \sim 1$ in the Hubble Deep Field North, which gives encouraging results for applications to large samples of high-redshift galaxies. This method of morphological classification could be used to study the evolution of the morphology with redshift and is expected to put observational constraints on scenarios of galaxy evolution.
\end{abstract}

Key words. galaxies: fundamental parameters - galaxies: high-redshift - galaxies: evolution - galaxies: stellar content ultraviolet: galaxies

\section{Introduction}

The morphology of galaxies is the result of physical processes that have acted on these systems since their formation. In an attempt to organize galaxies and to understand their evolution, the Hubble-Sandage classification, based on the bulge-to-disk ratio, was based on the study of a sample of nearby bright galaxies (Sandage 1961). However, it now appears that this classification is not appropriate for faint nearby galaxies or small objects. Moreover, with telescopes reaching out to redshifts $z \geq 1$ the proportion of unclassified galaxies seems to increase with the distance, and a large fraction has an unusual morphology (Brinchmann et al. 1998; Abraham et al. 1996; van den Bergh 2002). The origin of this morphological change with $z$ is likely twofold: the morphological K-correction (Burgarella et al. 2001) and intrinsic galaxy evolution (Giavalisco et al. 1996). A first step in working out a method of morphological classification is to classify galaxies within morphological boxes and to calibrate the method where the results can be checked by eye, i.e. in the nearby universe. We also want to discriminate between merging and irregular galaxies, in order to confirm the validity of galaxy evolution models. The Hubble classification fails on this point
(Naim \& Lahav 1997). By comparing morphological characteristics of local and distant samples it might be possible to constrain the mechanisms of formation and evolution of galaxies in the early universe. As a result, it is necessary to work out a new system of classification, which should be more objective than the Hubble classification. A scheme based on quantitative indices would have the advantage of not implicating human judgment and to be suitable for automatization, in order to classify quickly large samples of galaxies. The quantification also allows us to measure the variation of the morphology with wavelength $(\lambda)$. Finally, for comparing low- and high-redshift galaxies these parameters have to be robust against degradation of spatial resolution and low signal-to-noise ratios $(S / N)$.

In the course of the years, a number of parameters has been proposed. Morgan $(1958,1959)$ originally used the index of light concentration in the center of the galaxy - the basis of the Hubble scheme with the morphology of the spiral structure. Kent (1985) and Abraham et al. (1994) developed two quantitative measures of concentration. Abraham et al. (1996) also used an asymmetry index of galaxies developed by Schade et al. (1995). The resulting asymmetry-concentration diagram calibrated from a local sample allowed them to distinguish three morphological types in the visible: E/SO galaxies, 
Table 1. Data used for the calibration of the method. (1): Marcum et al. (2001). CTIO: Cerro Tololo Inter-American Observatory. MLO: Mount Laguna Observatory; (2): Larsen \& Richtler (1999), Danish 1.54 m, La Silla, 0.4"'pixel; (3): Kuchinski et al. 2000; (4): Boselli et al. (private communication). OHP: Observatoire de Haute-Provence, 0.69"/pixel; (5): James et al. (2003). JKT: Jacobus Kapteyn Telescope, la Palma; (6): Palomar P60; 0.378"/pixel; Madore et al. (private communication). (7): Schmidt telescope $1 \mathrm{~m}$; 2.5"/pixel; Lauberts \& Valentijn (1989); (8): Milliard et al. (1992).

\begin{tabular}{|c|c|c|c|c|c|c|c|c|c|c|}
\hline Galaxy & $1500 \AA$ & $2000 \AA$ & $2500 \AA$ & $U$ & $B$ & $V$ & $R$ & $I$ & UV data & Optical data \\
\hline NGC 628 & & & $\mathrm{X}$ & & & & $\mathrm{X}$ & & UIT (1) & P60 (6) \\
\hline NGC 1313 & $\mathrm{X}$ & & & $\mathrm{X}$ & $\mathrm{X}$ & $\mathrm{X}$ & $\mathrm{X}$ & $\mathrm{X}$ & UIT (1) & Danish $1.54 \mathrm{~m}$ (2) \\
\hline NGC 1316 & & & $\mathrm{X}$ & & & $\mathrm{X}$ & $\mathrm{X}$ & & UIT (1) & CTIO (1) \\
\hline NGC 1317 & & & $\mathrm{X}$ & & $X$ & & $\mathrm{X}$ & & UIT (1) & CTIO (1) \\
\hline NGC 1399 & $\mathrm{X}$ & & $\mathrm{X}$ & & $\mathrm{X}$ & & $\mathrm{X}$ & & UIT (1) & MLO (1) \\
\hline NGC 1404 & $\mathrm{X}$ & & $\mathrm{X}$ & & $\mathrm{X}$ & & $\mathrm{X}$ & & UIT (1) & ESO archive (7) \\
\hline NGC 2403 & $\mathrm{X}$ & & & $\mathrm{X}$ & $\mathrm{X}$ & $\mathrm{X}$ & $\mathrm{X}$ & & UIT (1) & P60 (3) \\
\hline NGC 2903 & $\mathrm{X}$ & & & $\mathrm{X}$ & $\mathrm{X}$ & $X$ & $\mathrm{X}$ & & UIT (1) & P60 (3) \\
\hline NGC 2993 & $\mathrm{X}$ & & $\mathrm{X}$ & & & & $\mathrm{X}$ & & UIT (1) & MLO (1) \\
\hline NGC 3031 & & $\mathrm{X}$ & $\mathrm{X}$ & & $X$ & $\mathrm{X}$ & $\mathrm{X}$ & & FOCA (8) and UIT (1) & OHP (4) \\
\hline NGC 3034 & & & $\mathrm{X}$ & $\mathrm{X}$ & $\mathrm{X}$ & & $\mathrm{X}$ & & UIT (1) & OHP (4) \\
\hline NGC 3310 & $\mathrm{X}$ & & & & & & $\mathrm{X}$ & & UIT (1) & JKT (5) \\
\hline NGC 3351 & $\mathrm{X}$ & & & & $\mathrm{X}$ & $\mathrm{X}$ & $\mathrm{X}$ & $\mathrm{X}$ & UIT (1) & P60 (3) \\
\hline NGC 3389 & $\mathrm{X}$ & & & & & $\mathrm{X}$ & & & UIT (1) & P60 (3) \\
\hline NGC 4038/39 & $\mathrm{X}$ & & & & & & $\mathrm{X}$ & & UIT (1) & ESO archive (7) \\
\hline NGC 4214 & $\mathrm{X}$ & & & & $\mathrm{X}$ & $\mathrm{X}$ & $\mathrm{X}$ & $\mathrm{X}$ & UIT (1) & P60 (3) \\
\hline NGC 4321 & & $\mathrm{X}$ & & $X$ & $\mathrm{X}$ & $\mathrm{X}$ & $\mathrm{X}$ & & FOCA (8) & OHP (4) \\
\hline NGC 4449 & $X$ & & & & $\mathrm{X}$ & $X$ & $\mathrm{X}$ & $\mathrm{X}$ & UIT (1) & P60 (3) \\
\hline NGC 4736 & $\mathrm{X}$ & & & $\mathrm{X}$ & & & $\mathrm{X}$ & & UIT (1) & P60 (3) \\
\hline NGC 5055 & $\mathrm{X}$ & & & $\mathrm{X}$ & $\mathrm{X}$ & $X$ & $\mathrm{X}$ & & UIT (1) & P60 (3) \\
\hline NGC 5194 & $\mathrm{X}$ & $\mathrm{X}$ & & & $\mathrm{X}$ & $\mathrm{X}$ & $\mathrm{X}$ & & UIT (1) and FOCA (8) & OHP (4) \\
\hline NGC 5236 & $\mathrm{X}$ & & & $\mathrm{X}$ & $\mathrm{X}$ & & $\mathrm{X}$ & $\mathrm{X}$ & UIT (1) & CTIO (3) \\
\hline
\end{tabular}

spiral galaxies and irregular/peculiar galaxies (Abraham et al. 1996; Bershady et al. 2000). Moreover, it seems possible to discriminate irregular galaxies from mergers by using a correlation between color index and asymmetry (Conselice et al. 2000).

Kuchinski et al. (2000) and Burgarella et al. (2001) compared rest-frame UV images with images in the visible and made pioneering studies where they showed how asymmetry and concentration evolve with wavelength for several galaxies. Therefore we must take care to compare objects that are actually comparable. More specifically, we must understand the behavior of the concentration and the asymmetry as a function of wavelength before comparing results from samples at different $z$. Finally, a physical interpretation of the morphology and its evolution with wavelength is necessary to understand the formation and the evolution of galaxies through high- $z$ observations, and to constrain models.

This work relies on the largest good-quality data sample presently available from UV to visible wavelengths. The paper aims at describing how to define a spectro-morphology classification from the local sample to $z \sim 1$ galaxies. It outlines the main parameters and analyses their relative strengths and weaknesses.

The paper is organized as follows: the data are presented in Sect. 2. We discuss the concentration and asymmetry calculations in Sect. 3. Section 4 presents the results of multi- $\lambda$ measurements. We calibrate these parameters from the nearby sample; and in Sect. 4.1 we give an example of application at high $z$. Finally, we present in Sect. 5 our conclusions and perspectives.

\section{Data}

\subsection{Sample selection}

A preliminary task is to gather the largest multi- $\lambda$ sample of nearby galaxies from UV to $I$-band. The sample of galaxies is listed in Table 1. The main constraint on building a multiwavelength sample is the availability of UV data. The sample is strongly biased against early-type galaxies, which are underrepresented because they are too faint in the UV. We used all the UV data in the local universe available to build a sample weighted toward spiral and irregular galaxies and toward nonactive galaxies.

The UV data at $1500 \AA$ and $2500 \AA$ mainly come from the Ultraviolet Imaging Telescope (UIT, Marcum et al. 2001), which has a pixel size of $1.14^{\prime \prime} /$ pixel and a $F W H M$ of about $3.3^{\prime \prime}$. We have complemented the UV sample with data from the FOCA telescope (Milliard et al. 1992), which were observed at $2000 \AA$ with a pixel scale of $3.44^{\prime \prime} /$ pixel and $F W H M=12^{\prime \prime}$ for M 100; a pixel size of $5.2^{\prime \prime} /$ pixel and $F W H M=20^{\prime \prime}$ for $\mathrm{M} 81$. 
The pixel scales for optical images range from $0.35^{\prime \prime} /$ pixel to $1.40^{\prime \prime} /$ pixel in the visible with a spatial resolution varying from $1.5^{\prime \prime}$ to $3.3^{\prime \prime}$. The origins of the images are listed in Table 1 . The physical properties of these galaxies are listed in Table 2 and are illustrated in Fig. 2.

\section{Morphological parameters}

The photometry was performed using the IRAF/ELLIPSE software. The apertures were centered on the highest-intensity pixel of the galaxies. The ellipse parameters for each galaxy were estimated by fitting the isophote at $3 \sigma_{\text {noise }}$ over the background.

\subsection{Surface brightness}

Bershady et al. (2000) defined a total photometry aperture using the local surface brightness. More specifically they introduced a non-dimensional parameter $\eta$, as the ratio between the local surface brightness at the distance $r$ and the average surface brightness within $r: \eta(r)=I(r) /<I(r)>$. They defined the total photometry aperture as twice the semi-major axis where $\eta(r)=0.2$. The total flux measured at this radius is similar to that estimated using the curve of growth. Since it is based on a ratio of two fluxes, $\eta$ is not very sensitive to the total luminosity of the galaxy, which allows us to use it on lowluminosity galaxies. According to Bershady et al. (2000), for an exponential profile more than $99 \%$ of the flux is included within $r(\eta=0.2)$, and about $89 \%$ for an $r^{1 / 4}$-law profile, because of the slower decrease at large radii. For comparison, the ratio between $r(\eta=0.2)$ and the half-light radius is 2.16 and 1.82 respectively for exponential and $r^{1 / 4}$-law profiles. In the following we adopt the method of Bershady et al. to estimate the total photometry aperture of galaxies.

\subsection{Concentration of light}

Kent (1985) defined a parameter of concentration $(C)$ based on the ratio of two isophotal radii: the first contains $80 \%$ of the total flux of the galaxy, the second contains $20 \%$ of that flux. Kent's parameter is expressed as:

$C=5 \log \left(\frac{r_{80 \%}}{r_{20 \%}}\right)$.

The inner and outer radii are chosen in order to optimize the dynamical range of concentration and to be sensitive enough to the variations of morphological types. Theoretically, a $r^{\frac{1}{4}}$-law profile corresponds to a concentration value $C=5.2$, and an exponential profile to $C=2.7$. Bershady et al. (2000) showed that $C$ is very stable against a spatial resolution degradation and thus appears to be robust parameter for the study of high-redshift galaxies. We simulated images by degrading the signal-to noise ratio and the spatial resolution: the mean error in $C$ is estimated to be $\Delta C \sim 0.2$. Hereafter we adopt $C$ as our parameter of concentration.

\subsection{Asymmetry}

\subsubsection{Definition}

The asymmetry index was first defined by Schade et al. (1995):

$A_{S}=\frac{1}{2} \frac{\sum\left|I_{0}-I_{180^{\circ}}\right|}{\sum I_{0}}$

as a measure of the asymmetry of galaxies to a $180^{\circ}$ rotation. It consists in computing the pixel by pixel difference between the original image and its $180^{\circ}$ rotation. In the expression of $A_{S}$, $I_{0}$ is the intensity for a given pixel and $I_{180^{\circ}}$ is the intensity of its rotationally-symmetric counterpart. The sky background is subtracted and careful flat-fielding must be performed before any calculation. The rotation centre is the position that yields a minimum value for the asymmetry.

Kuchinski et al. (2000) computed the asymmetry only from the pixels above a threshold of light intensity, in order to cancel the effect of pixels that do not contain galaxy light. Indeed, the number of those pixels within the aperture can be important, particularly in UV images of galaxies, which often have a patchy appearance. We used the following definition of asymmetry, inspired by Kuchinski et al. (2000):

$A=\frac{1}{2} \frac{\sum I>n \sigma_{\text {noise }}\left|I-I_{0}\right|}{\sum I_{0}}$

where $n \sigma_{\text {noise }}$ is the threshold above the sky background. We chose $n=2$.

A correction term must be added to the asymmetry, to remove the background asymmetry. Abraham et al. (1996) and Conselice et al. (2000) proposed to select a patch of sky with the same size as the aperture to compute its asymmetry. They used the following correction term:

$A_{\text {sky }}=\frac{1}{2} \frac{\sum\left|B_{0}-B_{180^{\circ}}\right|}{\sum I_{0}}$

where $B_{0}$ and $B_{180^{\circ}}$ are the intensities of a given pixel onthe sky and its rotationally-symmetric counterpart. However, this method requires having enough sky in the image around the galaxy; and the time of computation is rather long. We try to avoid these constraints by assuming that the sky noise is purely poissonian, and calculate the noise asymmetry in a statistical way:

$\sum\left|B_{0}-B_{180^{\circ}}\right|=\frac{2}{\sqrt{\pi}} \sigma_{\text {sky }} N_{\text {pix }}$.

We tested that the sky noise asymmetry we obtained by the statistical way is very close to the values obtained with the method of Abraham et al. (1996) and Conselice et al. (2000). Depending on the quality of images, the uncertainty in the sky noise asymmetry, $\Delta A_{\text {noise }}$, can vary from \pm 0.001 to \pm 0.01 .

Hereafter we adopt the following definition of the asymmetry:

$A=\frac{1}{2}\left[\frac{\sum I>n \sigma_{\text {noise }}\left|I-I_{0}\right|}{\sum I_{0}}-k_{\text {scale }} \frac{2}{\sqrt{\pi}} \frac{\sigma_{\text {noise }} N_{\text {pix }}}{\sum I_{0}}\right]$ 
Table 2. Concentration and asymmetry parameters and their variations with wavelength. ${ }^{1}$ See http: //leda. univ-lyon 1 . fr. ${ }^{2}$ Morphological type from the RC $3 .{ }^{3}$ Computed by interpolation when NUV data are not available, because of the significant changes of $A$ and $C$ between $1500 \AA$ and $3500 \AA .{ }^{4}$ Value in the $R$-band, in ".

\begin{tabular}{|c|c|c|c|c|c|c|c|c|c|}
\hline Galaxy & Type $t^{1}$ & RC3 type ${ }^{2}$ & Physical peculiarities & $A(R)$ & $C(R)$ & $A(U V)-A(R)^{3}$ & $C(U V)-C(R)^{3}$ & $r(\eta=0.2)_{R}{ }^{4}$ & $M_{B}{ }^{1}$ \\
\hline NGC 628/M $74 \ldots$ & 5.2 & $\mathrm{SA}(\mathrm{s}) \mathrm{c}$ & $\ldots$ & 0.051 & 2.84 & 0.12 & -0.83 & 212 & -20.29 \\
\hline NGC 1313 & 6.9 & $\mathrm{SB}(\mathrm{s}) \mathrm{d}$ & $\ldots$ & 0.145 & 2.97 & 0,152 & $-0,23$ & 232 & -19.12 \\
\hline NGC $1316 \ldots \ldots$ & -1.9 & $\mathrm{SAB}(\mathrm{s}) 0$ & pec & 0.028 & 4.42 & 0,033 & $-0,63$ & 268 & -22.07 \\
\hline NGC $1317 \ldots \ldots$ & 0.7 & $\mathrm{SAB}(\mathrm{r}) \mathrm{a}$ & ring UV & -0.003 & 4.64 & 0,043 & $-3,01$ & 66 & -20.16 \\
\hline NGC 1399 & -4.9 & E1 & pec & -0.034 & 2.92 & 0,077 & 0,32 & 168 & -20.95 \\
\hline NGC $1404 \ldots \ldots$ & -5 & E1 & $\ldots$ & -0.018 & 2.99 & 0,049 & 0,08 & 132 & -21.13 \\
\hline NGC $2403 \ldots \ldots$ & 5.8 & $\mathrm{SAB}(\mathrm{s}) \mathrm{cd}$ & $\ldots$ & 0.081 & 2.61 & 0,206 & $-0,20$ & 316 & -19.52 \\
\hline NGC 2903 & 4 & $\mathrm{SAB}(\mathrm{rs}) \mathrm{bc}$ & sbrst & 0.090 & 3.00 & 0,146 & $-0,03$ & 230 & -20.90 \\
\hline NGC $2993 \ldots . .$. & 1.1 & $\mathrm{Sa}$ & pec, sbrst & 0.097 & 3.97 & $-0,028$ & $-0,58$ & 25 & -19.72 \\
\hline NGC 3031/M 81 & 2.2 & $\mathrm{SA}(\mathrm{s}) \mathrm{ab}$ & ring UV, LINER, Sy 1.8 & 0.036 & 4.06 & 0,154 & -2.21 & 457 & -21.54 \\
\hline NGC 3034/M 82 & 10 & I0 & $\ldots$ & 0.115 & 2.62 & 0.137 & -0.25 & 173 & -18.52 \\
\hline NGC $3310 \ldots \ldots$ & 4 & $\mathrm{SAB}(\mathrm{r}) \mathrm{bc}$ & pec, sbrst & 0.140 & 3.47 & $-0,016$ & $-0,56$ & 30 & -20.25 \\
\hline NGC 3351/M 95 & 2.9 & $\mathrm{SB}(\mathrm{r}) \mathrm{b}$ & ring UV, sbrst & 0.023 & 3.98 & $\ldots$ & 0,65 & 166 & -20.30 \\
\hline NGC $3389 \ldots \ldots$ & 2.3 & $\mathrm{SA}(\mathrm{s}) \mathrm{c}$ & $\ldots$ & 0.138 & 2.56 & 0,041 & $-0,49$ & 76 & -19.79 \\
\hline NGC $4038 / 9 \ldots$ & 99 & merger & $\ldots$ & 0.299 & 2.04 & 0.145 & -0.36 & 165 & $-21.34 /-21.30$ \\
\hline NGC 4214 & 10 & $\mathrm{IAB}(\mathrm{s}) \mathrm{m}$ & $\ldots$ & 0.085 & 3.30 & 0,206 & 0,63 & 153 & -17.11 \\
\hline NGC 4321/M 100 & 4.3 & $\mathrm{SAB}(\mathrm{s}) \mathrm{bc}$ & $\ldots$ & 0.047 & 2.94 & $\ldots$ & $-0,57$ & 190 & -22.12 \\
\hline NGC $4449 \ldots \ldots$ & 10 & $\mathrm{IBm}$ & $\ldots$ & 0.112 & 2.90 & 0,151 & $-0,05$ & 127 & -17.80 \\
\hline NGC 4736/M 94 & 2.1 & (R)SA(r)ab & LINER, Sy 2 & 0.000 & 4.07 & 0,105 & $-1,61$ & 49 & -21.06 \\
\hline NGC 5055/M 63 & 4 & $\mathrm{SA}(\mathrm{rs}) \mathrm{bc}$ & $\ldots$ & 0.043 & 3.48 & 0,072 & $-0,75$ & 293 & -21.22 \\
\hline NGC 5194/M 51 & 4.2 & $\mathrm{SA}(\mathrm{s}) \mathrm{bc}$ & pec, Sy 2.5 & 0.095 & 3.14 & 0,136 & $-1,06$ & 221 & -19.70 \\
\hline NGC 5236/M 83 & 5.1 & $\mathrm{SAB}(\mathrm{s}) \mathrm{c}$ & sbrst & 0.040 & 3.01 & 0,181 & 0,08 & 353 & -21.12 \\
\hline
\end{tabular}


where

- $N_{\text {pix }}$ is the number of pixels within the aperture;

- $k_{\text {scale }}$ is a scaling factor equal to the ratio of the number of pixels used for computing $A$ to the number of pixels in the patch of sky.

We compared our values of asymmetry to the values obtained by Conselice et al. (2000) when studying images in $B$-band of a sample of 113 nearby bright galaxies (Frei et al. 1996). From this comparison we estimated the uncertainty in $A$ to be of the order of $\Delta A= \pm 0.02$.

\subsubsection{Reliability of the asymmetry index}

The asymmetry index mainly depends on two factors: the spatial resolution and the signal-to-noise ratio per pixel $S / N_{\text {pix }}$. Conselice et al. (2000) defined a minimum spatial resolution that is linked to the size of star-forming regions. They introduced $\epsilon=\frac{\theta_{0.5 \mathrm{kpc}}}{\theta_{\mathrm{res}}}$, the ratio of the angular size of $0.5 h_{75}^{-1} \mathrm{kpc}$ in the galaxy to the spatial resolution. They showed by degrading the pixel scale that below $\epsilon \sim 1$ asymmetry values fall, galaxies becoming too symmetric: morphological details such as star-forming regions are not resolved anymore. Nevertheless Conselice et al. (2000) estimated that for large galaxies a resolution of $1 \mathrm{kpc}$ is acceptable. Indeed, some acceptable values of $A$ found for images at $\epsilon<1$ (e.g. M 100 in UV taken by FOCA with $\epsilon \simeq 0.6$ ) suggest that this constraint on spatial resolution is not absolute. We verified that for all the images except the FOCA image of M 100 the condition defined by Conselice et al. (2000) is satisfied.

Once the physical constraint on the spatial resolution is secured, the other decisive factor, of a statistical nature, is $S / N_{\text {pix }}$. In order to test its effect we degraded $S / N_{\text {pix }}$ for several galaxies of various types and various wavelengths, previously resized to the same physical scale: $\epsilon \sim 2.7$ one pixel covers about $0.2 \mathrm{kpc}$ in the galaxy. The asymmetry shows low changes as long as $S / N_{\text {pix }} \geq 1$ (Fig. 1). When $S / N_{\text {pix }} \leq 1$, large diffuse regions of the galaxy become invisible. Only the brightest structures are seen; because of the selection of the brightest pixels, few are used in the computation of $A$. As a result, $A$ increases. Therefore we must have $S / N_{\text {pix }} \geq 1$ to compute $A$ safely. This statistical condition is valid whatever the pixel scale if the condition for the spatial resolution $\epsilon \geq 1$ is satisfied. All the data compiled (except NGC 3351 at $1500 \AA$ which was not used for asymmetry computation) satisfied the physical constraint on the spatial resolution and the statistical condition on $S / N_{\text {pix }}$, i.e. $\epsilon \geq 1$ and $S / N_{\text {pix }} \geq 1$.

\section{Results}

\subsection{Bandshifting effects}

Measuring the variation of asymmetry and concentration indices as a function of $\lambda$ is equivalent to observing the distribution of different types of stellar populations emitting at different wavelengths within a galaxy. Thus we might be able to distinguish different types of galaxies. Star-forming regions and young blue stars are prominent in the rest-frame (RF) UV whereas older stellar population are observed in the RF visible. Consequently, at these two wavelengths there could be a large change in the morphology of galaxies with two stellar population components, like spiral galaxies. For the same galaxy, concentration values in the UV are expected to be lower than in the optical (Kuchinski et al. 2000). Indeed, some early spiral galaxies, NGC 1317, M 81, and M 94, present a ring of young stars in the UV (Reichen et al. 1994; Waller et al. 2001), with a faint or nonexistent central bulge that trends to decrease the concentration value. In the $R$-band, they are very concentrated because of their prominent central bulge of old red stars. Thus, this significant change in concentration is due to the coexistence of two stellar populations that are distributed differently. The change in concentration is less dramatic for late-type galaxies: their star-forming regions are more or less uniformly distributed in over the galaxy, and their surface brightness profiles are well fitted by an exponential profile. Irregular galaxies like NGC 4449 and M 82 have a constant concentration in UV and visible, which is the expression of their having only one young stellar component.

The clumpy appearance of late-type spirals in the UV, due to the presence of star-forming regions, gives higher values of $A$ in the UV than in optical wavelengths, where the disk appears smoother because of a more uniform distribution of old stars.

\subsection{Spectro-morphological types}

\subsubsection{Qualitative definitions}

Previous work (Abraham et al. 1996; Bershady et al. 2000) showed that it is possible to discriminate three main morphological types of galaxies (E/SO, spiral and irregular/merger) from an asymmetry-concentration diagram built in the $R$ or $B$-band. This is not possible in UV, as shown by Burgarella et al. (2001) and Kuchinski et al. (2000): at these wavelengths, spiral galaxies appear to be of a later type and we are not able to distinguish them from irregular galaxies. Therefore one-band classifications only use part of the available information and would lead to misinterpretations at high redshift $(z \geq 1)$. A multi-wavelength study would allow us to better define morphological types which would take into account some elements of physics of galaxies, such as distribution and proportion of stellar populations and star-forming activity.

Figure 3 shows the asymmetry and concentration parameters of the galaxies in our multi-band sample as a function of wavelength. Five generic types of behaviors were observed, which we will hereafter call spectro-morphological types:

- Compact galaxies: these galaxies are characterized by a low asymmetry value whatever the wavelength; and globally high values of concentration, with little change with wavelength. This spectro-morphological type includes $\mathrm{E} / \mathrm{SO}$ galaxies, and early spiral galaxies dominated by their central bulge at all wavelengths. That there is little change in asymmetry and concentration is the result of the presence of only one stellar component in these galaxies. 


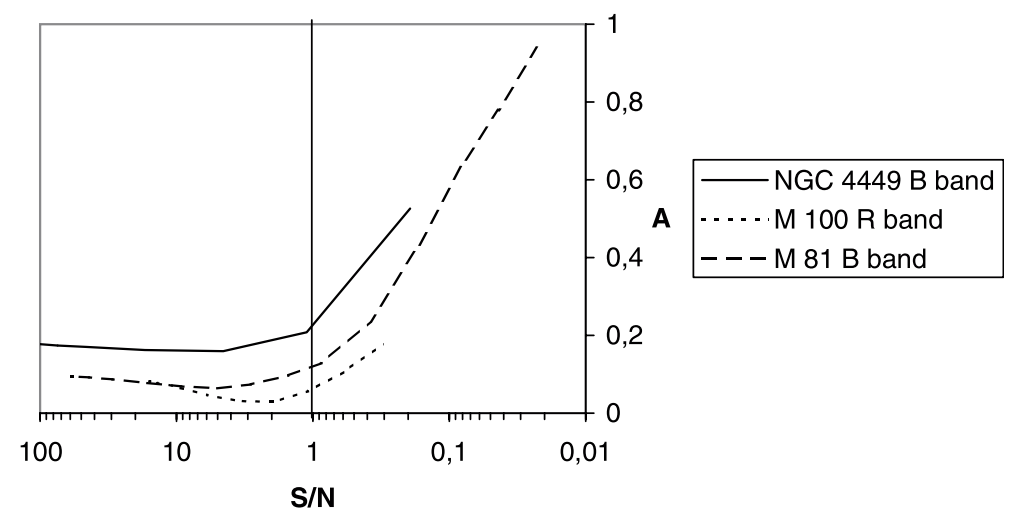

Fig. 1. Variations of $A$ as a function of the signal-to-noise per pixel within $r(\eta=0.2)$. There is a conservative limit at $S / N_{\text {pix }}=1$ above which the method can be safely applied. One pixel covers about $0.2 \mathrm{kpc}$.

- Ringed galaxies: these galaxies feature in the UV a faint or non-existent central bulge surrounded by a bright ring of stellar formation activity. This ring is often induced by orbital resonance with the central bulge or a stellar bar, and likely takes place at the inner Lindblad resonance of these galaxies (e.g. NGC 4736). Rings might also be due to past merger activity or gravitational interaction. Ringed galaxies show a prominent central bulge of old stars at optical wavelengths. This dramatic difference in the distribution of young and old stars yields large changes with wavelength in the concentration. The change in asymmetry is not very significant because of the symmetry of the ring structure.

- Spiral galaxies: their changes in concentration and asymmetry are the result of the existence of a composite stellar population. However, their appearance changes less dramatically with wavelength than that of ringed galaxie; they keep their spiral-like structure at all wavelengths. Star-forming regions are located in the spiral arms and give a patchy appearance in the UV whereas old stars form a prominent bulge and a uniform disk.

- Irregular galaxies: the main feature of these galaxies is the zero change in concentration with wavelength; the expected concentration value is close to 2.7 i.e. the theoretical value for an exponential surface-brightness profile. These galaxies do not show a prominent bulge either in the UV or in the visible; their main stellar component is young. Their patchy appearance in the UV gives high asymmetry values at this wavelength, which decrease toward longer wavelengths because of the more diffuse structure in the optical bands. Late spiral and irregular galaxies, with low surface brightness or flocculent disk are not distinguished and the two together constitute the irregular spectro-morphological type.

- Central starburst galaxies: galaxies with a central starburst are easily recognizable from their particularly high concentration value in $U V$, and a positive change in concentration from the UV to the $R$-band. As suggested by Colina et al. (1997) and Scoville (2003), there may be a strong link between nuclear starburst activity and the presence of an AGN. We expect to find some active galaxies in this class.

\subsubsection{Spectro-morphological boxes}

Quantitatively, we are able to define spectro-morphological boxes, based on $A(R), C(R)$, and their changes with wavelength, $\Delta A$ and $\Delta C$ (Table 3). The elliptical galaxies NGC 1399 and NGC 1404, which are not very concentrated, may not be very representative of this morphological class. A larger sample of early galaxies is necessary to improve the analysis, but we expect the general trends to be true. Each spectro-morphological type is characterized by a distinct shift in the asymmetryconcentration diagram when studied in the visible and UV. We illustrate this effect in Fig. 4a by plotting one typical case for each spectro-morphological type. For the sake of comparison, we also report the locus of the merger NGC 4038/9. Its changes in concentration and asymmetry with wavelength are the result of the existence of a composite stellar population. The high values of the asymmetry whatever the wavelength allow us to distinguish it from single systems. Larger samples will be necessary to generalize these conclusions behavior for mergers. Figure $4 \mathrm{~b}$ also shows $\Delta A$ versus $\Delta C$ for our sample. This spectro-morphological diagram is a projection of two of the four parameters $A(R), C(R), \Delta A$ and $\Delta C$ that define a spectromorphological type. We can empirically define five areas in this diagram, corresponding to the five spectro-morphological types defined above. In summary, when $\Delta C>0$, galaxies are classified as central-starburst galaxies. When $\sqrt{\Delta A^{2}+\Delta C^{2}}<1$, galaxies are classified as compact galaxies. UV-ringed galaxies, spiral galaxies and irregular galaxies areas are delimited by the following equations: $\Delta A=-0.31 \Delta C$ and $\Delta A=-0.088 \Delta C$, whith $\Delta C<0$.

\subsection{First application to high-z galaxies and perspectives for future research}

The application of spectro-morphology to high- $z$ galaxies can make use of deep surveys which provide multi- $\lambda$ data. As an example, we have computed $A$ and $C$ of three obviously spiral galaxies from the HDF (Bunker et al. 2000; Fig. 5): HDF 4-378, 4-474 and 4-550, respectively situated at $z=$ $1.2, z=1.059$ and $z=1.012$ and observed by HST at six wavelengths, from the RF FUV to the I-band. F300W, $F 450 W, F 606 W$ and $F 814 W$-filter images were provided by 

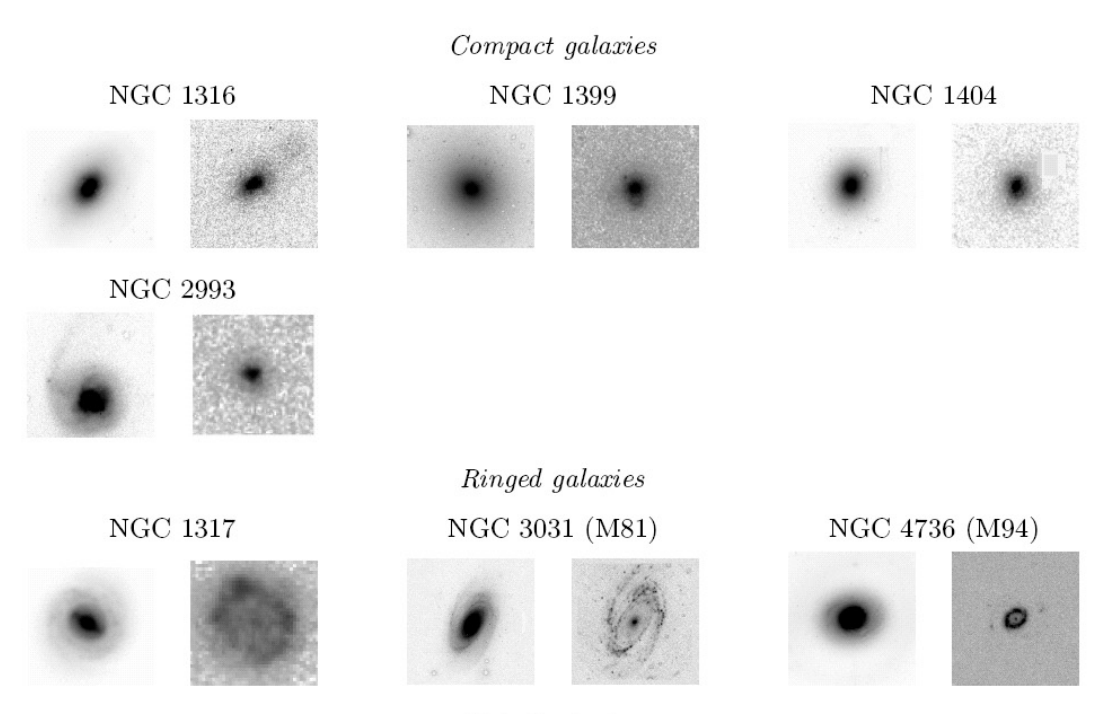

Ringed galaxies
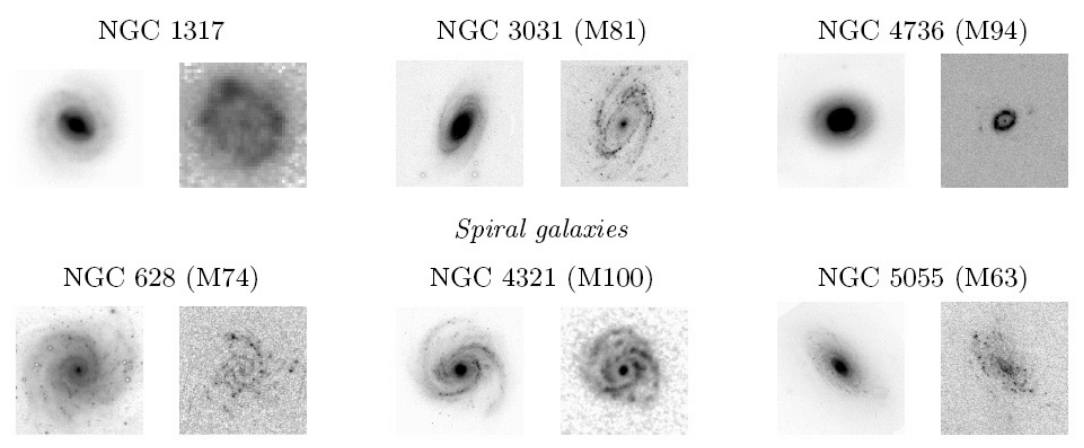

NGC 4321 (M100)
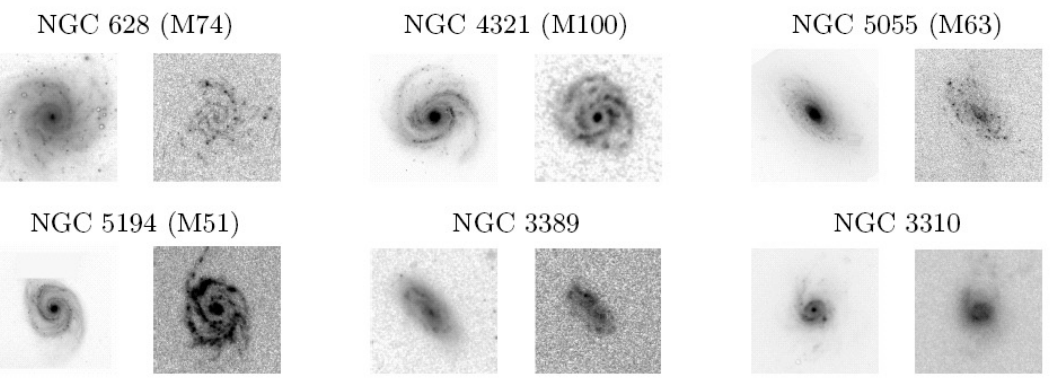

NGC 3389

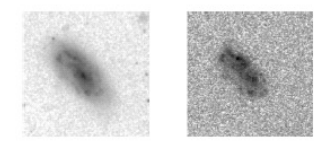

NGC 3310

Irregular-like galaxies

NGC 1313

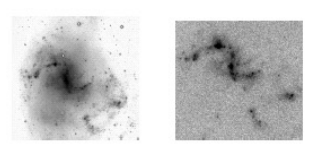

NGC 4449

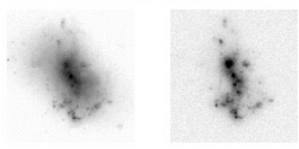

NGC 3351

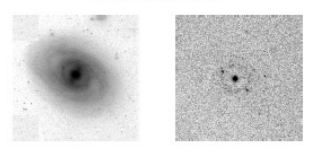

NGC 2903
Central starburst galaxies

NGC 4214

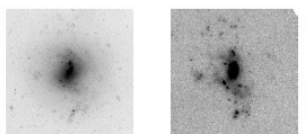

NGC 5236 (M83)

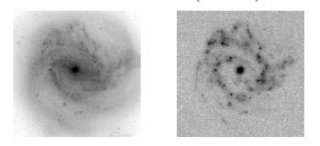

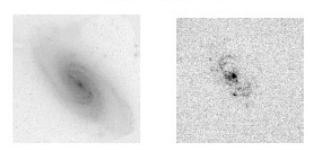
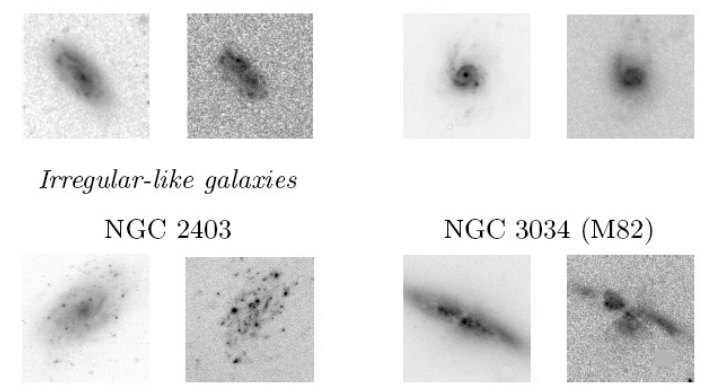

Fig. 2. Galaxies of our sample classified as a function of their spectro-morphological type. For each galaxy: left: optical image; right: UV image.

the HST Wide-Field Camera 2 with a pixel size of $0.045^{\prime \prime}$, i.e. $\epsilon \simeq 1.3 \mathrm{~F} 110 \mathrm{~W}$ and $\mathrm{F} 160 \mathrm{~W}$-filter images provided by NICMOS (NIC3) have a pixel size of $0.096^{\prime \prime}$, i.e. $\epsilon \simeq 0.7$. The values of $\epsilon$ for the NIC3 images are only slightly lower than the threshold recommended by Conselice et al. (2000). As noticed in Sect. 3.3 this threshold is not absolute, and the values of $A$ found with the NIC3 images are considered acceptable. We used the F450W, F606W, F814W, F110W and F160W images which correspond respectively to the RF near$\mathrm{UV}, U, B, V$ and $I$-bands. The signal-to-noise ratio per pixel of each image is larger than 1 . The changes in $C$ and $A$ (Table 4) found for HDF 4-378 and 4-474 are typically those found for 

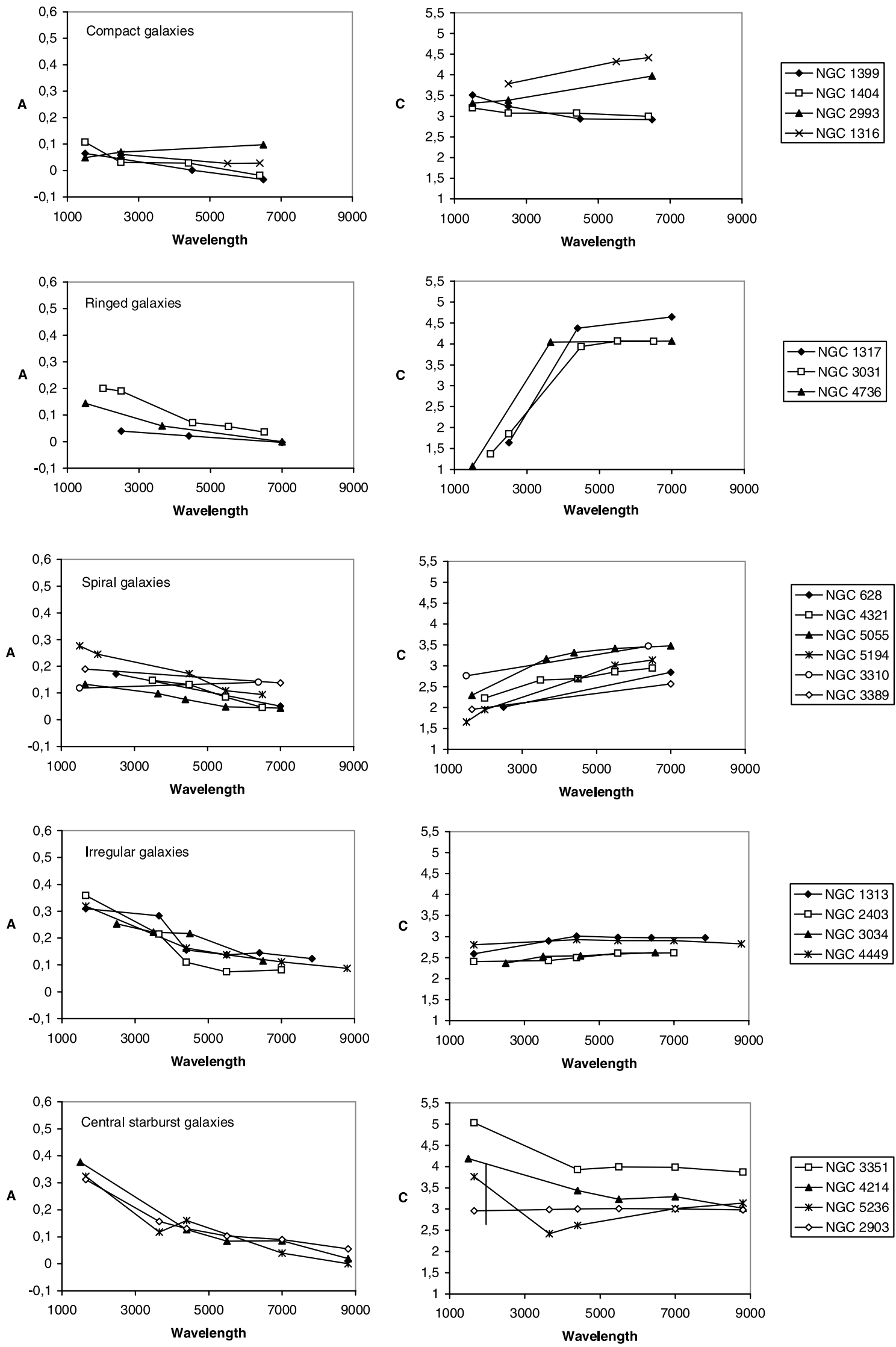

Fig. 3. Variations of concentration and asymmetry versus $\lambda$ in $\AA$. 
Table 3. Mean values of morphological parameters. ${ }^{1}$ Computed in the $2500 \AA$ and $6500 \AA$ rest frame and by interpolation if these wavelengths are not available. NGC 4038/9 is given as an example of a merging system.

\begin{tabular}{lcccc}
\hline \hline Morphological type & $A(R)$ & $C(R)$ & $A(N U V)-A(R)^{1}$ & $C(N U V)-C(R)^{1}$ \\
\hline Compact galaxies $\ldots \ldots$. & $0.018 \pm 0.059$ & $3.58 \pm 0.74$ & $0.033 \pm 0.045$ & $-0.20 \pm 0.48$ \\
Ringed galaxies $\ldots \ldots \ldots$ & $0.011 \pm 0.022$ & $4.26 \pm 0.33$ & $0.101 \pm 0.056$ & $-2.28 \pm 0.70$ \\
Spiral galaxies $\ldots \ldots \ldots$. & $0.086 \pm 0.046$ & $3.07 \pm 0.36$ & $0.090 \pm 0.054$ & $-0.75 \pm 0.20$ \\
Irregular galaxies ...... & $0.113 \pm 0.026$ & $2.78 \pm 0.19$ & $0.161 \pm 0.030$ & $-0.18 \pm 0.09$ \\
Central starburst galaxies & $0.059 \pm 0.033$ & $3.32 \pm 0.46$ & $0.160 \pm 0.046$ & $+0.33 \pm 0.36$ \\
\hline NGC 4038/9 & 0.299 & 2.04 & 0.145 & -0.36 \\
\hline
\end{tabular}

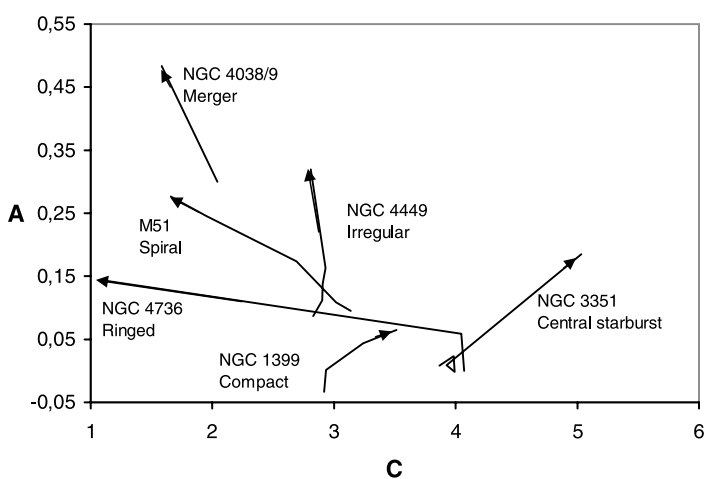

(a)

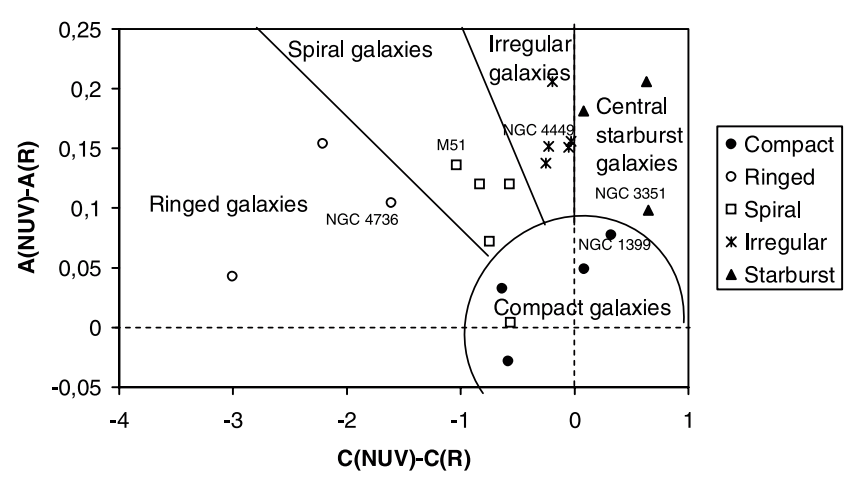

(b)

Fig. 4. a): Shift of some representative galaxies of each spectro-morphological type from $R$-band to UV. The arrows point toward the UV. The location of NGC 4038/9 on the diagram is away from the location of single systems. b): Change in $C$ versus change in $A$. It is possible to discriminate five spectro-morphological areas: compact, ringed, spiral, irregular and central starburst.

Table 4. Morphological parameters computed for three high- $z$ galaxies. ${ }^{1}$ Taken from Fernández-Soto et al. (1999). ${ }^{2}$ Rest-frame wavelength. ${ }^{3}$ Computed in the $2500 \AA$ and $6500 \AA$ rest frame and by interpolation if these wavelengths are not available.

\begin{tabular}{lccccc}
\hline \hline Object & Spectral type $^{1}$ & $A(R)^{2}$ & $C(R)^{2}$ & $A(N U V)-A(R)^{2,3}$ & $C(N U V)-C(R)^{2,3}$ \\
\hline HDF 4-378 $\ldots$ & Irregular & 0,066 & 2,34 & 0,141 & $-0,63$ \\
HDF 4-474 $\ldots$ & Irregular & 0,056 & 2,22 & 0,177 & $-0,82$ \\
HDF 4-550 $\ldots$ & Scd & 0,064 & 2,50 & 0,332 & $-0,89$ \\
\hline
\end{tabular}

spiral galaxies, whereas both galaxies were classified as irregular by Fernández-Soto et al. (1999) from their spectral energy distributions (SEDs). For HDF 4-550, the change in asymmetry is quite high, due to the presence of particularly bright star-forming regions in the arms of that galaxy. The larger the changes in $C$ and in $A$ with the wavelength, the more dramatic the contrast in the distribution of young and old stellar populations. This is an example of successful application to high-redshift galaxies. However, we must understand the influence of active nuclear and interacting objects, which we expect to be more important at larger distances. AGN galaxies would likely be classified as central starburst galaxies, because of the central activity that often occurs in the central bulge (Maiolino et al. 1999). Moreover, we have to focus on the application of spectro-morphology to merger systems, expected to be more numerous at high redshift. One of the main advantages of this method would be if it could help us to avoid misclassifying spiral galaxies as irregulars. Indeed, high- $z$ observations often seem to suggest that there is an increase of irregular galaxies with redshift and a low contribution of spiral galaxies, which are strong constraints on models of galaxy formation. So, it is important to discriminate spiral from irregular galaxies. A multi- $\lambda$ view will make it possible to use all the information contained in the images and minimize misclassifications of spirals as irregulars.

A more detailed study of high-redshift galaxies is deferred to a next paper. Interacting systems and AGN galaxies, inducing star formation activity at the centre of these galaxies, are expected to be more common than in the local universe. Spectro-morphology relies on stellar populations. By studying the distribution of stellar populations we could put observational constraints on the evolution scenarios of galaxies.

\section{Conclusion}

A multi-wavelength study of a sample of nearby objects allowed us to work out what we call spectro-morphology, i.e. the study of the change of asymmetry and concentration with wavelength. We saw that galaxies with two stellar components (spiral galaxies of the Hubble classification) present the highest 

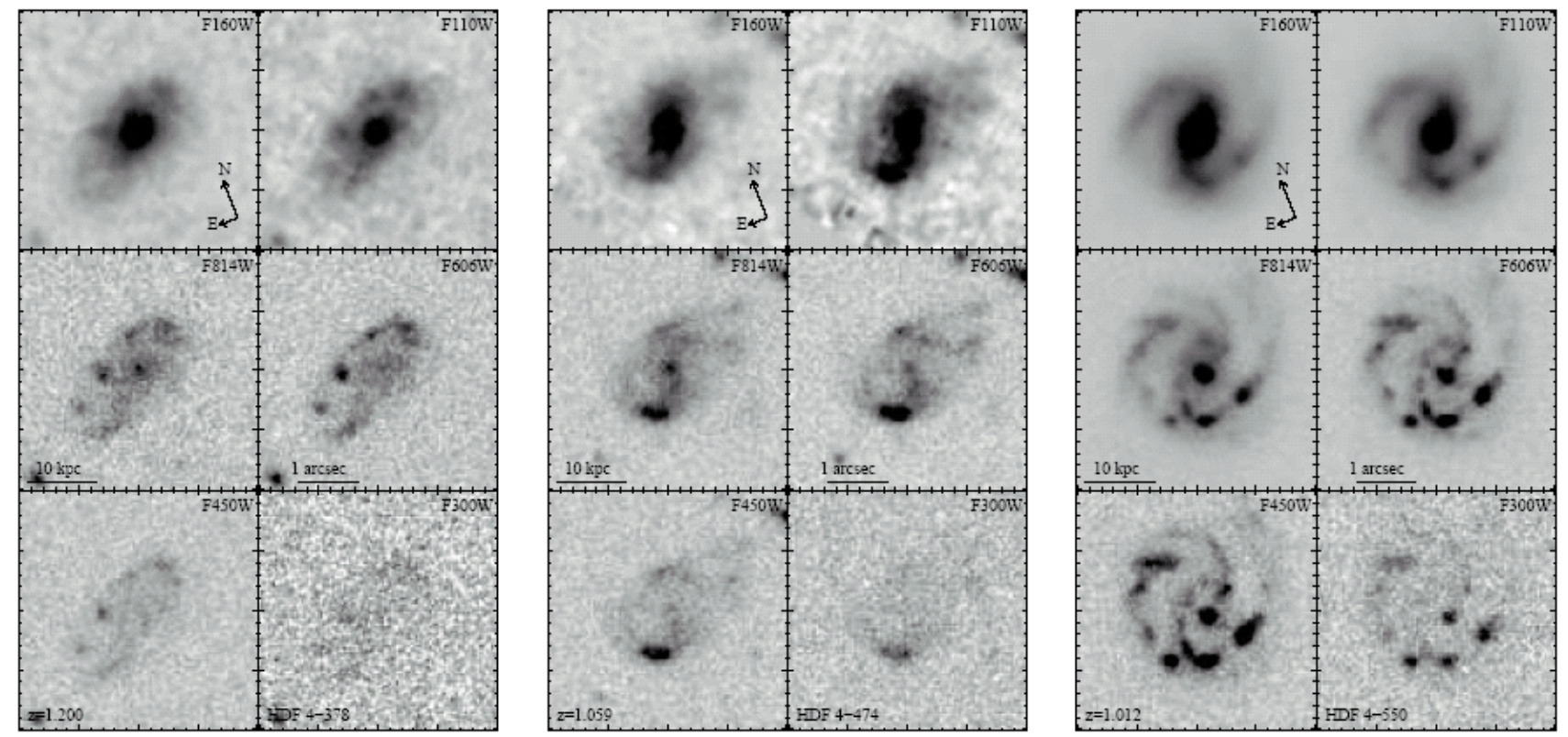

Fig. 5. Multi-wavelength images of the three galaxies HDF 4-378, 4-474 and 4-550. The F160W images (RF I-band) show a prominent bulge and the spiral structure of disk, at least for HDF 4-474 and 4-550. At shorter wavelengths star-forming regions appear. The $F 300 W$ images were removed from the study because of a too low signal-to-noise ratio.

variation of both indices, whereas the variation of galaxies with one stellar component (ellipticals/lenticulars and irregulars) are less wavelength-dependent. More precisely the changes in concentration and in asymmetry with wavelength between the UV and the $R$-band led us to define five spectro-morphological types, characterized by the distribution of their stellar populations. Compact galaxies are concentrated and symmetric at all wavelengths. This constancy of $C$ and $A$ is the result of the presence of only one stellar component. Ringed galaxies are often early spiral galaxies with a prominent bulge in the visible, which exhibit a ring of star-forming activity in the UV: the loci of young and old stellar populations are very different, which induces large changes in $C$ and $A$ from UV to optical bands. Spiral galaxies show a prominent bulge in the visible and starforming regions in the arms of the spiral structure in the UV. The resulting changes in $A$ and $C$ with wavelength are less dramatic than for ringed galaxies, but indicate the existence of a composite stellar population. Irregular galaxies have a concentration parameter close to 2.7 at all wavelengths, which is the value expected for a galactic disk, and are rather asymmetric. This class contains galaxies with a faint or non-existent central bulge, which in the Hubble classification are classified as late spiral or irregular. Their stellar population is dominated by young stars. Central-starburst galaxies possess a very bright nucleus in the UV that yields a high concentration. This starburst activity is often induced by gravitational interaction or the existence of an AGN. The correlation between $C(N U V)-C(R)$ and $A(N U V)-A(R)$ allowed us to identify these five spectromorphological types. To test the validity of our method at high redshift, we successfully applied it to three distant objects in the HDF at $z \sim 1$. The resulting morphology is consistent with an inspection by eye morphology and these galaxies are classified as spiral galaxies.
Acknowledgements. We thank Andrew Bunker for his collaboration, and Rodger I. Thompson for giving authorization to use the HDF multi-wavelength images. We are also grateful to the anonymous referee who largely contributed to improving this work. The support given by ASTROVIRTEL, a Project funded by the European Commission under FP5 Contract No. HPRI-CT-1999-00081 is acknowledged.

\section{References}

Abraham, R. G., Valdes, F., Yee, H. K. C., \& van den Bergh, S. 1994, ApJ, 432, 75

Abraham, R. G., van den Bergh, S., Glazebrook, K., et al. 1996, ApJS, 107,1

Baugh, C. M., Cole, S., \& Frenk, C. S. 1996, MNRAS, 283, 1361

Bershady, M. A., Jangren, A., \& Conselice, C. J. 2000, AJ, 119, 2645

Bresolin, F., \& Kennicutt, R. C. 2002, ApJ, 572, 838

Brinchmann, J., Abraham, R., Schade, D., et al. 1998, ApJ, 499, 112

Bunker, A., Spinrad, H., Stern, D., et al. 2000

[arXiv: astro-ph/0004348]

Burgarella, D., Buat, V., Donas, J., Milliard, B., \& Chapelon, S. 2001, A\&A, 369, 421

Buta, R. 1995, ApJS, 96, 39

Castellani, M., \& Tornambe, A. 1991, ApJ, 381, 393

Colina, L., Garcia Vargas, M. L., Mas-Hesse, J. M., Alberdi, A., \& Krabbe, A. 1997, ApJ, 484, L41

Conselice, C., Bershady, M. A., Dickinson, M., et al. 1998, BAAS, 30, 1368

Conselice, C. J., Bershady, M. A., \& Jangren, A. 2000, ApJ, 529, 886

de Vaucouleurs, G., de Vaucouleurs, A., Corwin, H. G., et al. 1991, Vol. 1-3, XII, 2069 (Berlin, Heidelberg, New York: Springer-Verlag)

Dirsch, B., Richtler, T., Geisler, D., et al. 2003, AJ, 125, 1908

Drozdovsky, I. O., Schulte-Ladbeck, R. E., Hopp, U., Greggio, L., \& Crone, M. M. 2002, AJ, 124, 811 
Elmegreen, D. M., Chromey, F. R., \& Warren, A. R. 1998, AJ, 116, 2834

Fernández-Soto, A., Lanzetta, K. M., \& Yahil, A. 1999, ApJ, 513, 34 J

Frei, Z., Guhathakurta, P., Gunn, J. E., \& Tyson, J. A. 1996, AJ, 111, 174

Giavalisco, M., Livio, M., Bohlin, R. C., Macchetto, F. D., \& Stecher, T. P. 1996, AJ, 112, 369

Greve, A., Wills, K. A., Neininger, N., \& Pedlar, A. 2002, A\&A, 383, 56

Harris, J., Calzetti, D., Gallagher, J. S., Conselice, C. J., \& Smith, D. A. 2001, AJ, 122, 3046

Hill, R. S., Fanelli, M. N., Smith, D. A., et al. 1998, ApJ, 507, 179

Horellou, C., Black, J. H., van Gorkom, J. H., et al. 2001, A\&A, 376, 837

James, P. A., Shane, N. S., Beckman, J. E., et al. 2003 [arXiv: astro-ph/0311030]

Kent, S. M. 1985, ApJS, 59, 115

Knapen, J. H., Beckman, J. E., Heller, C. H., Shlosman, I., \& de Jong, R. S. 1995, ApJ, 454, 623

Kuchinski, L. E., Madore, B. F., Freedman, W. L., \& Trewhella, M. 2000, ApJS, 131, 441

James, P. A., Shane, N. S., Beckman, J. E., et al. 2003 [arXiv: astro-ph/0311030]

Lamers, H. J. G. L. M., Panagia, N., Scuderi, S., et al. 2002, ApJ, 566, 818

Larsen, S. S., \& Richtler, T. 1999, A\&A, 345, 59

Lauberts, A., \& Valentijn, E. A. 1989, The surface photometry catalogue of the ESO-Uppsala galaxies (Garching: European Southern Observatory)

Maiolino, R., Alonso-Herrero, A., Anders, S., et al. 1999, Adv. Space Res., 23, 875

Marcum, P. M., O’Connell, R. W., Fanelli, M. N., et al. 2001, ApJS, 132,129
Milliard, B., Donas, J., Laget, M., Armand, C., \& Vuillemin, A. 1992, A\&A, 257, 24

Morgan, W. W. 1958, PASP, 70, 364

Morgan, W. W. 1959, PASP, 71, 92

Muñoz-Tuñón, C., Caon, N., \& Aguerri, J. A. L. 2004, AJ, 127, 58

Naim, A., \& Lahav, O. 1997, MNRAS, 286, 969

O’Connell, R. W., Bohlin, R. C., Collins, N. R., et al. 1992, ApJ, 395, L45

Papovich, C., Giavalisco, M., Dickinson, M., Conselice, C. J., \& Ferguson, H. C. 2003, ApJ, 598, 827

Reichen, M., Kaufman, M., Blecha, A., Golay, M., \& Huguenin, D. 1994, A\&AS, 106, 523

Ryder, S. D., Staveley-Smith, L., Malin, D., \& Walsh, W. 1995, AJ, 109, 1592

Sandage, A. 1961, The Hubble atlas of galaxies (Washington: Carnegie Institution)

Schade, D., Lilly, S. J., Crampton, D., et al. 1995, ApJ, 451, L1

Schweizer, F. 1980, ApJ, 237, 303

Scoville, N. 2003, J. Korean Astron. Soc., 36, 167

Seigar, M. S. 2002, A\&A, 393, 499

Sérsic, J. L. 1973, PASP, 85, 103

Smith, D. A., Neff, S. G., Bothun, G. D., et al. 1996, ApJ, 473, L21

Stecher, T. P., Cornett, R. H., Greason, M. R., et al. 1997, PASP, 109, 584

Stoughton, C., Lupton, R. H., Bernardini, M. A., et al. 2002, ApJ, 123, 485

Thornley, M. D., \& Mundy, L. G. 1997, ApJ, 484, 202

van den Bergh, S., Abraham, R. G., Ellis, R. S., et al. 1996, AJ, 112, 359

van den Bergh, S. 2002, PASP, 114, 797

Waller, W. H., Gurwell, M., \& Tamura, M. 1992, AJ, 104, 63

Waller, W. H., Fanelli, M. N., Keel, W. C., et al. 2001, AJ, 121, 1395 